\title{
MAGEC2 is a sensitive and novel marker for seminoma: a tissue microarray analysis of 325 testicular germ cell tumors
}

\author{
Peter K Bode ${ }^{*, 1}$, André Barghorn ${ }^{1, *}$, Florian R Fritzsche ${ }^{1}$, Marc-Oliver Riener ${ }^{1, \dagger}$, \\ Glen Kristiansen ${ }^{1}$, Alexander Knuth ${ }^{2}$ and Holger Moch ${ }^{1}$ \\ ${ }^{1}$ Institute of Surgical Pathology, University Hospital Zurich, Zurich, Switzerland and ${ }^{2}$ Clinic of Oncology, \\ University Hospital Zurich, Zurich, Switzerland
}

\begin{abstract}
Melanoma-associated gene C2 (MAGEC2) is a recently identified cancer testis antigen expressed in normal testicular and placental tissue. It has been detected in some human carcinomas, but its expression in primary testicular germ cell tumors is unknown. Immunohistochemistry was used to study MAGEC2 protein in 325 primary testicular germ cell tumors, including 94 mixed germ cell tumors. Seminomatous and nonseminomatous components were separately arranged and evaluated on tissue microarrays. MAGEC2 expression was compared with POU5F1 (OCT3/4), SOX2, SOX17, KIT and TNFRSF8 (CD30). The mouse monoclonal anti-MAGEC2 antibody (clone LX-CT10.5) revealed a nuclear MAGEC2 expression with little or no background staining. MAGEC2 expression was found in 238 of 254 seminomas (94\%), but not in embryonal carcinomas $(n=89)$. POU5F1 (OCT3/4) was positive in $97 \%$ of seminomas and all embryonal carcinomas. In contrast, KIT was positive in $94 \%$ of seminoma but also in $8 \%$ of embryonal carcinomas. TNFRSF8 (CD30) and SOX2 were negative in seminoma and positive in embryonal carcinoma (96 and 90\%, respectively). SOX17 was positive in $94 \%$ of seminoma and negative in embryonal carcinoma. We conclude that MAGEC2 allows a reliable distinction of seminoma from embryonal carcinomas. Therefore, MAGEC2 represents an additional tool for the differential diagnosis of testicular germ cell tumors.

Modern Pathology (2011) 24, 829-835; doi:10.1038/modpathol.2011.6; published online 4 March 2011
\end{abstract}

Keywords: cancer testis antigens; embryonal carcinoma; immunohistochemistry; POU5F1 (OCT3/4); SOX2; spermatogenesis

\section{Introduction}

Cancer testis antigens (CT antigens) are the product of germ line-associated genes that are present in normal germ line tissues, such as testis, placenta and ovary and in various human cancers. ${ }^{1-3}$ So far, more than 100 CT genes have been identified by different methods, including serological analysis of recombinant expression libraries. ${ }^{3}$ CT genes can be divided into those that are encoded on the $\mathrm{X}$-chromosome (CT-X antigens) and those that are

${ }^{*}$ Correspondence: Dr PK Bode, MD, Institute of Surgical Pathology, University Hospital Zurich, Schmelzbergstrasse 12, 8091 Zurich, Switzerland.

E-mail: PeterKarl.Bode@usz.ch

${ }^{*}$ Current address: Institute of Pathology Medica, Zurich, Switzerland.

Current address: Institute of Pathology, University Hospital Erlangen, Erlangen, Germany.

Received 14 April 2010; revised 30 November 2010; accepted 3 January 2011; published online 4 March 2011 not (non-X CT antigens). ${ }^{3}$ Among CT-X antigens, the melanoma-associated gene (MAGE) family is the largest. ${ }^{4}$ Although CT antigens have been implicated in normal tissue development and tumorigenesis of various tumors, their function remains largely unknown. In several different tumor types, eg, multiple myeloma, non-small cell lung cancer, bladder cancer, hepatocellular carcinoma, breast, colorectal and pancreatic ductal adenocarcinoma, the expression of $C T-X$ genes is associated with progressive disease, poor differentiation grade and worse prognosis. ${ }^{5-8}$ Recently, a novel CT-antigen, MAGEC2, also known as CT10, was identified. MAGEC2 has been isolated in a melanoma cell line (SK-MEL-37). ${ }^{9}$ Concerning the homology between different CT antigens, MAGEC2 seems strongly related to MAGEC1 (CT7) at cDNA and at genomic levels (both genes map in close proximity to chromosome Xq27), but rather different from MAGEA1 and MAGEB1. ${ }^{10}$ MAGEC2 mRNA expression was identified in melanomas and some 
human carcinoma types by RT-PCR in fresh frozen tissue..$^{9,11}$

Expression of CT antigens seems to be restricted to male germ cells in the normal testis. ${ }^{12}$ CT-X antigens are usually highly expressed during the early phases of spermatogenesis, especially in spermatogonia and primary spermatocytes, but not in spermatids. ${ }^{3}$ Therefore, it seems likely that CT-X antigens repress the expression of genes, which are necessary for cell differentiation.

There are multiple types of testicular germ cell tumors with different degrees of differentiation. Seminomas show the phenotypes of early germ cells and possess pluripotent potential. Embryonal carcinomas are also intrinsically pluripotent. Other germ cell tumor types, including yolk sac tumors, mature teratomas and choriocarcinomas, are more differentiated. ${ }^{13,14}$

Studies on CT antigen expression in testicular germ cell tumors are limited. MAGEA1, MAGEA2, MAGEA3 and MAGEA4 mRNA expression has been studied in 32 germ cell tumors and was seen in 68$82 \%$ of seminomas, mixed germ cell tumors with seminomatous components and some non-seminomatous mixed germ cell tumors. Cheville and Roche $^{15}$ analyzed 43 germ cell tumors and observed MAGEA1 and MAGEA3 protein expression only in $17-42 \%$ of seminomas, but not in non-seminomatous tumors. ${ }^{16}$ MAGEC2 mRNA expression was previously detected in four of five seminomas by RT-PCR in fresh frozen tumor tissue, ${ }^{11}$ but there is no information on MAGEC2 expression in testicular germ cell tumors at the protein level so far.

In our study, the expression pattern of MAGEC2 was investigated in more than 300 primary testicular germ cell tumors using immunohistochemistry on tissue microarrays. MAGEC2 expression was compared with POU5F1 (OCT3/4), SOX2, SOX17, KIT and TNFRSF8 (CD30) expression. POU5F1 (OCT3/4) is a transcription factor that has been detected in neoplastic germ cells with pluripotent potential, eg, seminomas, embryonal carcinomas and intratubular germ cell neoplasia, a precursor lesion for malignant germ cell tumors of the adult testis. SOX2 is also a transcription factor that maintains the 'stemness' of cells. ${ }^{17}$ RT-PCR studies recently showed that SOX17 was amplified in seminoma but not in embryonal carcinoma. ${ }^{18,19}$ This finding could also be confirmed at the protein level. ${ }^{18,20}$ TNFRSF8 (CD30) is frequently used to identify embryonal carcinoma components in mixed germ cell tumors, whereas KIT is a sensitive seminoma marker. ${ }^{14}$ We provide evidence that embryonal carcinomas are MAGEC2 negative and POU5F1 (OCT3/4) positive, whereas MAGEC2 is expressed in almost all seminomas.

\section{Materials and methods}

\section{Patients}

A total of 325 patients diagnosed with testicular germ cell tumors were retrieved from the files of the
Institute of Surgical Pathology of the University Hospital Zurich, Switzerland from 1990 to 2003. Tumors were classified according to the 2004 WHO Classification $^{21}$ by an experienced uropathologist $(A B)$. The series included a total of 94 mixed germ cell tumors (49 with and 45 without a seminomatous component), 4 spermatocytic seminomas, 207 classic seminomas, 19 pure embryonal carcinomas and 1 pure mature teratoma. The 94 cases of mixed germ cell tumors included the following components: seminoma, embryonal carcinoma, yolk sac tumor, choriocarcinoma and teratoma.

Intratubular germ cell neoplasia unclassified (IGCNU) was included from 20 patients with testicular germ cell neoplasia. IGCNU was identified morphologically on hematoxylin- and eosin-stained histological section by virtue of seminoma-like cells with clear cytoplasm and enlarged nucleoli along the basal aspect of seminiferous tubules lacking normal spermatogenesis. Furthermore, the diagnosis was confirmed by immunohistochemical stainings for KIT, POU5F1 (OCT3/4) and placental alkaline phosphatase.

The project has been approved by the local ethics committee (reference number StV 25-2008).

\section{Tissue Microarray}

A tissue microarray was constructed as described before. ${ }^{5}$ Formalin-fixed paraffin-embedded tissue from the tumors was used according to tissue availability. All tumor types were represented by two tissue cores (diameter $0.6 \mathrm{~mm}$ ). If more than one tumor type was present in one patient (mixed germ cell tumors), each tumor type was separately punched and represented on the tissue microarray. In summary, the tissue microarray contained testicular tumor components of 254 seminomas, 89 embryonal carcinomas, 51 yolk sac tumors, 54 teratomas, 10 choriocarcinomas and 4 spermatocytic seminomas. In addition, we included non-tumor testicular tissue from 20 tumor patients. IGCNU was included from 20 patients (40 tissue cores). Thereby our tissue microarray comprised 1030 tumor tissue cores. During processing nine cases (18 tissue cores) were lost. For the evaluation of the immunostaining, we considered 472 different histological tumor components, 20 IGCNU and 20 non-neoplastic testicular tissues.

\section{Histology and Immunohistochemistry}

Sections ( $3 \mu$ thick) of tissue microarray blocks of formalin-fixed, paraffin-embedded tissues were mounted on glass slides (SuperFrost Plus; Menzel, Braunschweig, Germany), deparaffinized, rehydrated and stained with hematoxylin and eosin using standard histological techniques. For the detection of the MAGEC2 protein, a monoclonal antibody (clone LX-CT10.5, dilution 1:300; ref. 10) 
was used. The antibody was kindly provided by the Ludwig Institute for Cancer Research, New York. The dilution of the antibody was adjusted to the BOND Autostainer Medite system after performing titrations. HRP-DAB was used as chromogen. Immunoreactivity was nuclear for MAGEC2. Strong staining of spermatogonia in non-neoplastic testicular tissue served as normal controls. For the POU5F1 (OCT3/4) staining, a commercially available monoclonal antibody (clone N1NK, 1:150, Novocastra) was used. The dilution of the antibody was adjusted to the BOND Autostainer Medite system after performing titrations. HRP-DAB was used as chromogen. The immunohistochemical stainings for TNFRSF8 (CD30) (Dako M0751, Clone Her-H2, dilution 1:20), KIT (Dako A4502, polyclonal, dilution 1:100), SOX2 (Epitomics EPR3131, dilution 1:100) and SOX17 (OriGene Technologies, Clone 2G8, dilution 1:2000) were performed with the Ventana Benchmark automated staining system (Ventana Medical Systems, Tucson, AZ, USA) using Ventana UltraView DAB reagents. All primary antibodies were diluted in Ventana diluent. Slides were counterstained with hematoxylin, dehydrated and mounted.

The whole-tissue microarray was digitalized and evaluated using imaging software (Spotbrowser, Alphelys, Plaisir, France). An experienced surgical pathologist $(\mathrm{PKB})$ evaluated all tissue microarray spots on the computer monitor. Samples were dichotomized into positive vs negative for MAGEC2 and POU5F1 (OCT3/4). The threshold for positivity was defined at $5 \%$ of cells positive for the marker, as previously described. ${ }^{1}$ The expression patterns were separately analyzed for each tumor component, preneoplastic lesion and non-neoplastic tissue.

\section{Results}

MAGEC2 Antigen Expression in Neoplastic and NonNeoplastic Testicular Tissues

Immunohistochemical expression of MAGEC2 was easy to evaluate with a distinct nuclear staining. Stromal cells, Sertoli cells and Leydig cells were negative in all cases. In non-neoplastic germ cells, MAGEC2 showed a consistent and strong nuclear expression in $100 \%$ of cases. The staining was very strong in immature forms (spermatogonia and spermatocytes) and decreased gradually to more mature forms like spermatids. In sperms, no MAGEC2 expression could be demonstrated. Likewise, MAGEC2 was expressed in all cases (100\%) of IGCNU with a similar pattern as in non-neoplastic intratubular germ cells. In seminomas, MAGEC2 was identified in 238 of 254 cases (94\% Figure 1a and b; Table 1). In comparison with non-neoplastic germ cells and IGCNU, the intensity of the MAGEC2 expression in seminomas was generally little less pronounced. However, the positive cases showed the marker almost always in more than $50 \%$ of the cells. Interestingly, all four spermatocytic seminomas showed a strong CT10 immunostaining, similar to that in normal immature germ cells. From the other germ cell tumors components included in our analysis, 5 of 51 yolk sac tumors (10\%) and 1 of 10 choriocarcinomas (10\%) were positive for MAGEC2. Although the only positive choriocarcinoma showed a rather strong specific staining, merely single cells stained positive for MAGEC2 in the yolk sac tumors. None of the embryonal carcinomas or teratomas showed any nuclear expression of MAGEC2. Syncytiotrophoblastic cells stained always negative.

\section{POU5F1 (OCT3/4) Expression in Neoplastic and Non-Neoplastic Testicular Tissues}

Immunohistochemical expression of POU5F1 (OCT3/4) was also easy to evaluate with a nuclear staining. In stromal cells, Sertoli cells, Leydig cells and non-neoplastic germ cells, the POU5F1 (OCT3/4) staining was negative. In contrast POU5F1 (OCT3/4) was expressed in all cases (100\%) of IGCNU. In seminomas, POU5F1 (OCT3/4) could be detected in 246 of 254 cases (97\%). Interestingly, all four spermatocytic seminomas were negative. From the other germ cell tumors components included in our analysis all embryonal carcinomas showed a positive POU5F1 (OCT3/4) staining, whereas the remaining tumor components (yolk sac tumors, choriocarcinomas and teratomas) were negative.

\section{TNFRSF8 (CD30) Expression in Neoplastic and Non-Neoplastic Testicular Tissues}

Immunohistochemical expression of TNFRSF8 (CD30) showed a membranous staining pattern with a variable but overall quite strong intensity. In nonneoplastic germ cells, the TNFRSF8 (CD30) staining was negative. A total of 85/89 (96\%) embryonal carcinoma showed a positive staining pattern. All other tumor components were negative.

\section{KIT Expression in Neoplastic and Non-Neoplastic Testicular Tissues}

The immunohistochemical staining for KIT showed a membranous signal with overall strong intensity. Non-neoplastic germ cells were negative for KIT, whereas all cases of IGCNU were positive. A total of 239/254 (94\%) classic seminomas and all spermatocytic seminomas expressed KIT. Also 7/89 (8\%) embryonal carcinomas and 15/51 (30\%) yolk sac tumor showed a positivity for KIT. All remaining tumor components (choriocarcinomas and teratomas) were consistently negative.

\section{SOX2 Expression in Neoplastic and Non-Neoplastic Testicular Tissues}

The location of staining was nuclear with weak to moderate intensity. Immunohistochemical 
a
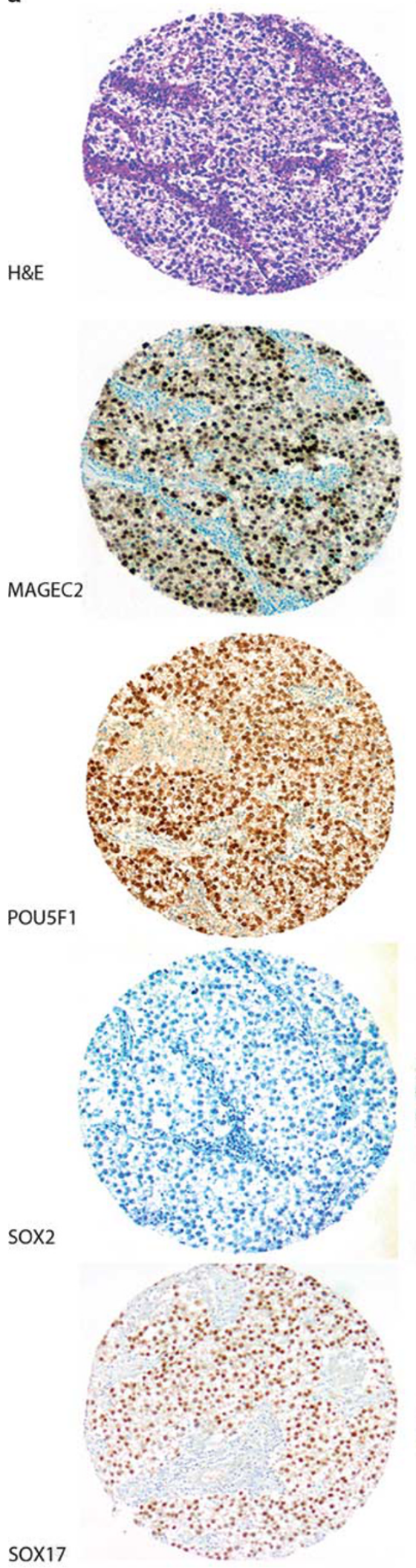

b
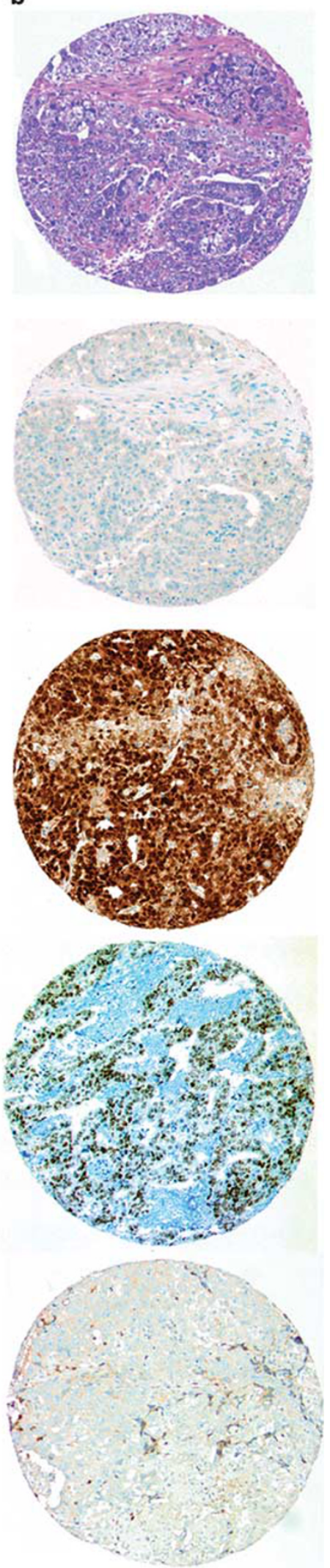

Figure 1 Corresponding conventional and immunhistochemical stains of seminoma and embryonal carcinoma. (a) Seminoma: hematoxylin and eosin (H\&E) staining; strong nuclear staining for MAGEC2 and POU5F1 (OCT3/4); negativity for SOX2 and positivity for SOX17. (b) Embryonal carcinoma: H\&E staining; negative staining for MAGEC2 and strong nuclear and cytoplasmic staining for POU5F1 (OCT3/4), moderate nuclear staining for SOX2 and negative staining for SOX17 (right). 
Table 1 MAGEC2 expression in non-neoplastic and neoplastic testicular tissues compared with other germ cell tumor markers

\begin{tabular}{|c|c|c|c|c|c|c|c|}
\hline Histology & No. of cases & MAGEC2 & KIT & POU5F1 (OCT3/4) & TNFRSF8 (CD30) & SOX2 & SOX17 \\
\hline Seminoma & 254 & $238(94 \%)$ & $239(94 \%)$ & $246(97 \%)$ & Negative & Negative & $238(94 \%)$ \\
\hline Sperm. seminoma & 4 & $4(100 \%)$ & $4(100 \%)$ & Negative & Negative & Negative & Negative \\
\hline Embryonal carcinoma & 89 & Negative & $7(8 \%)$ & $89(100 \%)$ & $85(96 \%)$ & $80(90 \%)$ & Negative \\
\hline Yolk sac tumor & 51 & $5(10 \%)$ & $15(30 \%)$ & Negative & Negative & Negative & $16(31 \%)$ \\
\hline Teratoma & 54 & Negative & Negative & Negative & Negative & $11(20 \%)$ & Negative \\
\hline Choriocarcinoma & 10 & $1(10 \%)$ & Negative & Negative & Negative & Negative & Negative \\
\hline IGCNU & 20 & $20(100 \%)$ & $20(100 \%)$ & $20(100 \%)$ & Negative & Negative & $20(100 \%)$ \\
\hline Germ cells & 20 & $20(100 \%)$ & Negative & Negative & Negative & Negative & Negative \\
\hline
\end{tabular}

Abbreviations: IGCNU, intratubular germ cell neoplasia, unclassified; MAGEC2, melanoma-associated gene C2.

expression of SOX2 was positive in $80 / 89(90 \%)$ embryonal carcinomas, but also in 11/56 (19.6) teratomas. All seminomas were uniformly negative as well as yolk sac tumor, choriocarcinoma, nonneoplastic germ cells and IGCNU.

\section{SOX17 Expression in Neoplastic and Non-Neoplastic Testicular Tissues}

SOX17 showed a nuclear expression with variable background staining. A total of 238/254 (94\%) classic seminomas expressed SOX17. All cases of IGCNU were positive. Also 16/51 (31\%) yolk sac tumor showed a positivity for SOX17. All remaining tumor components did not show a nuclear expression of SOX17.

\section{Discussion}

In this study of more than 300 testicular germ cell tumors, we identified MAGEC2 protein expression as a sensitive marker for seminomas, spermatocytic seminomas and IGCNU. The absence of MAGEC2 protein in embryonal carcinomas is of importance, because other 'seminoma marker', including KIT, placental alkaline phosphatase (PLAP) and POU5F1 (OCT3/4) are also expressed in embryonal carcinomas.

There is only little information on CT antigen expression in different types of testicular germ cell tumors. Yuasa et $a l^{2}$ and Hara et $a l^{16}$ analyzed different CT antigens in testicular tumors on mRNA level. CT mRNA expression was frequent in seminomas but rare in non-seminomatous germ cell tumors. On the protein level, CT antigens have only been reported in seminomas. Cheville and Roche ${ }^{15}$ have previously evaluated the expression of MAGEA1 and MAGEA3 in 43 germ cell tumors (24 seminomas, 6 embryonal carcinomas and 13 mixed germ cell tumors). Both proteins were detected in only $17 \%$ and $42 \%$, respectively, seminomas. Aubry et $\mathrm{al}^{22}$ investigated the expression of MAGEA4 in 27 germ cell tumors (17 seminomas and 10 nonseminomas). About one-third of the seminomas was negative. In contrast to other CT antigens, MAGEC2 stains almost all seminomas. Therefore, it is reasonable to predict that MAGEC2 may prove to be a valuable tool in the diagnosis of primary or metastatic seminoma.

For the detection of MAGEC2 protein, we used a previously generated monoclonal antibody (clone LX-CT10.5), applicable in formalin-fixed, paraffinembedded tissue. ${ }^{10}$ For CT-X antigens, strong nuclear and cytoplasmic staining in spermatogonia is usually observed. Therefore, testicular tissue can be used as positive control for antibodies against CT antigens. Immunohistochemical studies have shown MAGEA1 protein expression in the nucleus and cytoplasm of spermatogonia and primary spermatocytes. $^{12}$ Also GAGE1 and CTAG1B (NY-ESO-1) immunoreactivity is both nuclear and cytoplasmic, whereas MAGEC1 immunoreactivity is only cytoplasmic without nuclear expression. ${ }^{6,23}$ In contrast, the MAGEC2 antibody showed an exclusive nuclear staining pattern. The staining was very intense, distinct and with little background so that cells expressing MAGEC2 was unmistakable. This is consistent with the observation by Zhuang et $a{ }^{10}{ }^{10}$ who have shown that MAGEC2 protein expression is restricted to the nucleus and not to the cytoplasm.

Our finding of frequent MAGEC2 positivity in seminomas is consistent with findings of Lucas et al, ${ }^{11}$ who detected MAGEC2 mRNA in four of five seminomas by RT-PCR in fresh frozen tumor tissue. Lucas et $a l^{11}$ and Gure et $a l^{9}$ have also identified MAGEC2 mRNA expression in melanomas, bladder, breast, head and neck and non-small cell lung cancer. The tissue microarray approach in combination with antibodies for formalin-fixed, paraffinembedded tissue allows a high throughput determination of the prevalence of novel biomarkers. However, the tissue microarray approach may slightly underestimate the real prevalence of CT antigens, because CT antigen expression is characterized by a high level of intratumoral heterogeneity. $^{24}$ We have previously analyzed MAGEC2 and MAGEC1 protein expression in more than 200 liver tumors and observed focal protein expression in most CT antigen-positive tumors. ${ }^{5}$ We have seen an even higher rate of intratumoral heterogeneity for the CT antigen CTAG1B (NY-ESO-1) in breast 
cancer. ${ }^{6}$ In our testicular tumor microarray, all tumor types were represented by two tissue cores and different histological components of mixed germ cell tumors were analyzed separately. According to our data, there was always nuclear expression in more than $50 \%$ of the tumor cells in all tissue cores, suggesting a more homogeneous CT antigen expression in seminomas compared with other solid tumors.

IGCNU is a precursor lesion for malignant germ cell tumors and is frequently found adjacent to testicular germ cell tumors. Although MAGEC2 was expressed in all cases of IGCNU, it cannot serve as a diagnostic tool, because in the normal testis spermatogonia are also positive.

Our data provide evidence that MAGEC2 can be added to the current immunohistochemical markers for germ cell tumors, because of its unique staining pattern. POU5F1 (OCT3/4), KIT, PLAP, TNFRSF8 (CD30) (Ki-1) SOX2 and SOX17 are commonly used to accurately classify testicular tumors. Although POU5F1 (OCT3/4) and PLAP are positive in almost all seminomatous tumor components and in embryonal carcinomas, MAGEC2 is negative in all embryonal carcinomas. Similar results were found with SOX17 expression. However, spermatocytic seminomas were MAGEC2 positive, and SOX17 negative. Interestingly, SOX2 showed the opposite result compared with MAGEC2. Almost $90 \%$ of embryonal carcinoma showed an expression of SOX2, whereas all seminomas were negative for SOX2. Our tissue microarray results for SOX2 and SOX17 are consistent with results from other groups. ${ }^{17,18,20}$

The combination of several markers is often successful to determine the exact tumor components in mixed germ cell tumors. We feel that MAGEC2 constitutes a reasonable completion of this diagnostic armamentarium. In contrast to POU5F1 (OCT3/ 4), PLAP and KIT, MAGEC2 clearly helps in distinguishing seminomatous tumors from embryonal carcinoma or yolk sac tumors. Embryonal carcinoma is positive for the widely used seminoma-marker PLAP, whereas yolk sac tumors are often positive for KIT. ${ }^{13,25-27}$

Spermatocytic seminomas are clinically indolent tumors. They are adequately treated with orchiectomy alone and thus, need to be distinguished from classic seminoma, which may require adjuvant radiation, chemotherapy or surgery. The four cases of spermatocytic seminomas examined in this study were all positive for MAGEC2. Spermatocytic seminomas are largely negative for most other immunohistochemical markers, including PLAP, TNFRSF8 (CD30), POU5F1 (OCT3/4), SOX2, SOX17 and many others.

In summary, this is the first comprehensive analysis of MAGEC2 in testicular tumors. Our data strongly suggest MAGEC2 as an additional sensitive marker for the differentiation of seminomas from other types of germ cell tumors.

\section{Acknowledgements}

We thank M Storz, S Behnke and A Wethmar (Pathology Zurich) for excellent technical assistance. This work was supported by the Zurich Cancer League (Zurich) and the Cancer Research Institute.

\section{Disclosure/conflict of interest}

The authors declare no conflict of interest.

\section{References}

1 Jungbluth AA, Silva Jr WA, Iversen K, et al. Expression of cancer-testis (CT) antigens in placenta. Cancer Immun 2007;7:15

2 Yuasa T, Okamoto K, Kawakami T, et al. Expression patterns of cancer testis antigens in testicular germ cell tumors and adjacent testicular tissue. J Urol 2001;165:1790-1794.

3 Simpson AJ, Caballero OL, Jungbluth A, et al. Cancer/ testis antigens, gametogenesis and cancer. Nat Rev Cancer 2005;5:615-625.

4 van der Bruggen $\mathrm{P}$, Traversari C, Chomez P, et al. A gene encoding an antigen recognized by cytolytic $\mathrm{T}$ lymphocytes on a human melanoma. Science 1991;254:1643-1647.

5 Riener MO, Wild PJ, Soll C, et al. Frequent expression of the novel cancer testis antigen MAGE-C2/CT-10 in hepatocellular carcinoma. Int J Cancer 2009;124: 352-357.

6 Theurillat JP, Ingold F, Frei C, et al. NY-ESO-1 protein expression in primary breast carcinoma and metastases: correlation with CD8+ T-cell and CD79a+ plasmacytic/B-cell infiltration. Int $\mathrm{J}$ Cancer 2007;120:2411-2417.

7 Scanlan MJ, Gure AO, Jungbluth AA, et al. Cancer/ testis antigens: an expanding family of targets for cancer immunotherapy. Immunol Rev 2002;188:22-32.

8 Bergeron A, Picard V, LaRue $\mathrm{H}$, et al. High frequency of MAGE-A4 and MAGE-A9 expression in high-risk bladder cancer. Int J Cancer 2009;125:1365-1371.

9 Gure AO, Stockert E, Arden KC, et al. CT10: a new cancer-testis (CT) antigen homologous to CT7 and the MAGE family, identified by representational-difference analysis. Int J Cancer 2000;85:726-732.

10 Zhuang R, Zhu Y, Fang L, et al. Generation of monoclonal antibodies to cancer/testis (CT) antigen CT10/MAGE-C2. Cancer Immun 2006;6:7.

11 Lucas S, De Plaen E, Boon T. MAGE-B5, MAGE-B6, MAGE-C2, and MAGE-C3: four new members of the MAGE family with tumor-specific expression. Int J Cancer 2000;87:55-60.

12 Takahashi K, Shichijo S, Noguchi M, et al. Identification of MAGE-1 and MAGE-4 proteins in spermatogonia and primary spermatocytes of testis. Cancer Res 1995;55:3478-3482.

13 Oosterhuis JW, Looijenga LH. Testicular germ-cell tumours in a broader perspective. Nat Rev Cancer 2005;5:210-222.

14 Ulbright TM. Germ cell tumors of the gonads: a selective review emphasizing problems in differential diagnosis, newly appreciated, and controversial issues. Mod Pathol 2005;18(Suppl 2):S61-S79. 
15 Cheville JC, Roche PC. MAGE-1 and MAGE-3 tumor rejection antigens in human germ cell tumors. Mod Pathol 1999;12:974-978.

16 Hara I, Hara S, Miyake H, et al. Expression of MAGE genes in testicular germ cell tumors. Urology 1999;53:843-847.

17 Gopalan A, Dhall D, Olgac S, et al. Testicular mixed germ cell tumors: a morphological and immunohistochemical study using stem cell markers, POU5F1 (OCT3/4), SOX2 and GDF3, with emphasis on morphologically difficult-to-classify areas. Mod Pathol 2009;22:1066-1074.

18 de Jong J, Stoop H, Gillis AJ, et al. Differential expression of SOX17 and SOX2 in germ cells and stem cells has biological and clinical implications. J Pathol 2008;215:21-30.

19 Korkola JE, Houldsworth J, Chadalavada RS, et al. Down-regulation of stem cell genes, including those in a $200-\mathrm{kb}$ gene cluster at $12 \mathrm{p} 13.31$, is associated with in vivo differentiation of human male germ cell tumors. Cancer Res 2006;66:820-827.

20 Nonaka D. Differential expression of SOX2 and SOX17 in testicular germ cell tumors. Am J Clin Pathol 2009;131:731-736.
21 Eble JN, Sauter G, Epstein JE, et al. (eds). World Health Organization Classification of Tumours. Pathology and Genetics of Tumours of the Urinary System and Male Genital Organs. IARC Press: Lyon, 2004.

22 Aubry F, Satie AP, Rioux-Leclercq N, et al. MAGE-A4, a germ cell specific marker, is expressed differentially in testicular tumors. Cancer 2001;92:2778-2785.

23 Tinguely M, Jenni B, Knights A, et al. MAGE-C1/CT-7 expression in plasma cell myeloma: sub-cellular localization impacts on clinical outcome. Cancer Sci 2008;99:720-725.

24 Jungbluth AA, Stockert E, Chen YT, et al. Monoclonal antibody MA454 reveals a heterogeneous expression pattern of MAGE-1 antigen in formalin-fixed paraffin embedded lung tumours. Br J Cancer 2000;83:493-497.

25 Bahrami A, Ro JY, Ayala AG. An overview of testicular germ cell tumors. Arch Pathol Lab Med 2007;131: 1267-1280.

26 Biermann K, Klingmuller D, Koch A, et al. Diagnostic value of markers M2A, POU5F1 (OCT3/4), AP-2gamma, PLAP and c-KIT in the detection of extragonadal seminomas. Histopathology 2006;49:290-297.

27 Young RH. Testicular tumors-some new and a few perennial problems. Arch Pathol Lab Med 2008;132: 548-564. 